\title{
Comparison of two different measurement methods in evaluating basilar atherosclerotic plaque using high- resolution MRI at 3 tesla
}

\author{
Luguang Chen, Qian Zhan, Wenjia Peng, Tao Song, Qi Liu and Jianping Lu*
}

\begin{abstract}
Background: To compare the Self-referenced and Referenced measurement methods in assessing basilar artery (BA) atherosclerotic plaque employing dark blood high-resolution MRI at 3 Tesla.

Methods: Forty patients with $>20 \%$ stenosis as identified by conventional MRA were recruited and evaluated on a 3 Tesla MRI system. The outer wall, inner wall and lumen areas of maximal lumen narrowing site and the outer wall and lumen areas of sites that were proximal and distal to the maximal lumen narrowing site were manually traced. Plaque area (PA), stenosis rate (SR) and percent plaque burden (PPB) were calculated using the Self-referenced and Referenced measurement methods, respectively. To assess intra-observer reproducibility, BA plaque was measured twice with a 2-week interval in between measurements.
\end{abstract}

Results: Thirty-seven patients were included in the final analysis. There were no significant differences in PA, SR and PPB measurements between the two methods. The intra-class coefficients and coefficient of variations (CV) ranged from 0.976 to 0.990 and from 3.73 to $5.61 \%$ for the Self-referenced method and ranged from 0.928 to 0.971 and from 4.64 to $9.95 \%$ for the Referenced method, respectively. Both methods are effective in the evaluation of BA plaque. However, the CVs of the Self-referenced method is lower than the Referenced measurement method. Moreover, Bland-Altman plots showed that the Self-referenced method has a narrower interval than the Referenced measurement method.

Conclusions: The Self-referenced method is better and more convenient for evaluating BA plaque, and it may serve as a promising method for evaluation of basilar atherosclerotic plaque.

Keywords: Basilar artery, Atherosclerosis, Magnetic resonance imaging

\section{Background}

Atherosclerosis is a disease that progresses slowly and silently over decades, and the slow progress offers a chance for diagnosis before symptoms occur [1, 2]. Intracranial atherosclerosis is the most common reason for mortality in Asian populations [3]. The basilar artery (BA) is one of the largest intracranial arteries and is located in the posterior cerebral circulation. Basilar atherosclerotic plaque usually occurs in patients with ischaemic stroke and transient ischaemic attack (TIA)

* Correspondence: cjr.lujianping@vip.163.com

Department of Radiology, Changhai Hospital of Shanghai, The Second Military Medical University, No.168 Changhai Road, Shanghai 200433, China
[4]. Evaluation of morphologic characteristics of BA plaque (such as PA, plaque area; SR, stenosis rate and $\mathrm{PPB}$, percent plaque burden) is important and may guide treatment decisions in the clinical setting.

The rapid development of magnetic resonance imaging technology, especially at 3 Tesla field strength, offers a significant improvement in the signal-to-noise ratio, vessel wall to lumen contrast-to-noise ratio and image quality compared to imaging at 1.5 Tesla [5]. Dark blood high-resolution magnetic resonance imaging (HRMRI) has been demonstrated as a non-invasive and useful technique for evaluating the vessel wall in in vivo atherosclerotic disease [1]. It has been used to assess 
various major arteries in the whole body including the intracranial [6-10], carotid [11-15], coronary [16-18] and peripheral arteries [19-21] and the aorta [11, 22]. Accurate and fast assessment of the plaque burden and morphology of the basilar artery are paramount for determining treatment strategies for patients. Kim et al. used the maximal lumen narrowing (MLN) sites as the referenced sites to evaluate the degree of stenosis in basilar atherosclerotic plaque using HRMRI [23]. Previous studies have used the nearest plaque-free or minimally diseased segments proximal and distal to the MLN sites as the referenced sites to calculate morphologic parameters (herein referred to as the Referenced measurement method) [24-26]. In the present study, we have also employed the MLN sites as the referenced sites to compute the morphological parameters (herein referred to as the Self-referenced measurement method). Figure 1 shows the definitions of the Selfreferenced and Referenced measurement methods and their calculation rules for assessing BA plaque. However, studies on the comparison of the Self-referenced and Referenced measurement methods in assessing basilar atherosclerotic plaque have been limited. We hypothesized that the Self-referenced measurement method is better than the Referenced measurement method in evaluating plaque morphologic characterization. To the best of our knowledge, no such studies have been reported yet except our group [27].

Therefore, the goal of this study was to compare the Self-referenced and Referenced measurement methods in assessing basilar atherosclerotic plaque employing dark blood HRMRI at 3 Tesla.

\section{Methods}

\section{Subjects}

This prospective study was approved by the Committee on Ethics of Biomedical Research, Changhai Hospital of Shanghai, and written informed consent was obtained from each patient. Forty patients with $>20 \%$ stenosis as identified by conventional contrast-enhanced magnetic resonance angiography were recruited during the period from January 2014 to July 2016. The inclusion criteria were listed as follows: 1) Patients who had ischaemic stroke or TIA in the basilar artery territory within one month; 2) Occurrence of an ischaemic event in a vascular area that lies outside of the stenotic basilar artery; and 3) More than two risk factors for cardiovascular disease (e.g., hypertension, diabetes, smoking and hypercholesterolemia). The following criteria were used to exclude patients: 1) the degree of stenosis of the basilar artery was normal, occluded or $<20 \%$; 2) arteritis; 3) claustrophobia; and 4) poor image quality.

\section{Magnetic resonance imaging}

Cross-sectional imaging was performed on a 3 Tesla MRI system (MAGNETOM Skyra, Siemens medical solution, Erlangen, Germany) using a standard 20-channel phasedarray head/neck coil, with a peak gradient strength of 45 $\mathrm{mT} / \mathrm{m}$ and a slew rate of $200 \mathrm{mTm}^{-1} \mathrm{~ms}^{-1}$. Threedimensional time-of-flight magnetic resonance angiography (3D TOF-MRA) images were used for HRMRI image positioning and obtained with repetition time/echo time $(\mathrm{TR} / \mathrm{TE})=21 / 3.4 \mathrm{~ms}$, field of view $(\mathrm{FOV})=180 \times 200$ $\mathrm{mm}^{2}, \quad$ matrix $=330 \times 384$, thickness $=0.7 \mathrm{~mm}$, and

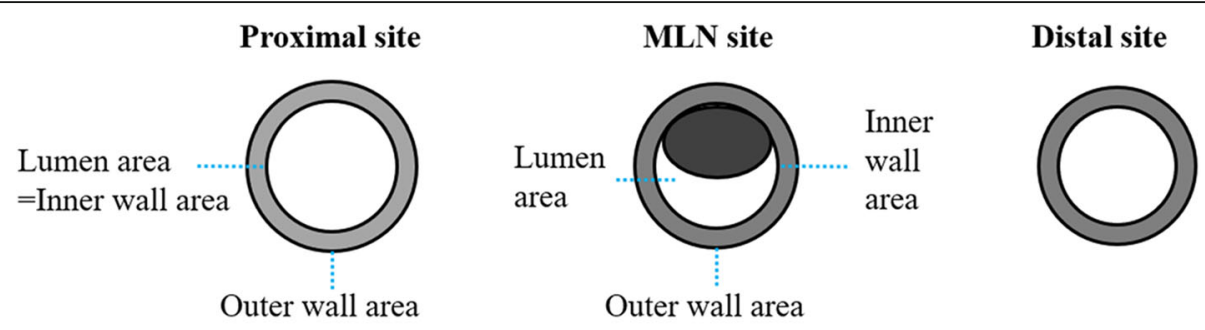

Self-referenced measurement method:

Plaque area $=$ Inner area at MLN site - Lumen area at MLN site

Stenosis rate $=$ Plaque area/Inner area at MLN site

Percent plaque burden $=$ Plaque area/Outer wall area at MLN site

Referenced measurement method:

Outer wall area at reference site $=$ average of the proximal and distal wall areas

Lumen area at reference site $=$ average of the proximal and distal lumen areas

Wall area at reference site $=$ Outer wall area at reference site - Lumen area at reference site

Wall area at MLN site $=$ Outer wall area at MLN site - Lumen area at MLN site

Plaque area $=$ Wall area at MLN site - Wall area at reference site

Stenosis rate $=1-$ Lumen area at MLN site/Wall area at reference site

Percent plaque burden $=$ Plaque area/Outer wall area at MLN site

Fig. 1 Definitions of the Self-referenced and Referenced measurement methods and their calculation rules for assessing BA plaque 
average $=1$, acquisition time $(\mathrm{TA})=4 \mathrm{~min} 40 \mathrm{~s}$. The main parameters of two-dimensional T2-weighted turbo spin echo (T2W TSE) were TR/TE $=2890 / 46 \mathrm{~ms}, \quad F O V=$ $100 \times 100 \mathrm{~mm}^{2}$, matrix $=256 \times 256$, thickness $=2 \mathrm{~mm}$, Gap $=0.5 \mathrm{~mm}$, echo train length $=20$, and averages $=2$, $\mathrm{TA}=3 \mathrm{~min} 40 \mathrm{~s}$. Fat saturation was applied to suppress signal from adjacent fatty tissues and improve identification of vessel wall boundaries. The black blood method with a regional saturation pulse of $60 \mathrm{~mm}$ thickness was employed to saturate the inflow arterial signal. The total scan time was approximately $8 \mathrm{~min}$.

\section{Image analysis}

Two experienced radiologists with 5 and 6 years of experience in vessel wall imaging and who were blinded to the clinical information of each patient assessed the image quality by consensus using 4-scale scores (score 1 , poor quality; score 2, adequate quality; score 3 , good quality; score 4, excellent quality) [28]. Images with a score of 1 were excluded from the final analysis. Quantitative measurement was carried out on images with a score of $\geq 2$. The outer wall, inner wall and lumen areas of the MLN site and the outer wall and lumen areas of the proximal and distal sites were manually traced using advanced image software (CMRTools, Cardiovascular Imaging Solutions, London, UK; Fig. 2). The calculation of PA, SR and PPB using the Referenced measurement method was carried out as per a previous study [24], which used the nearest normal segments proximal and distal to the MLN sites as the reference to calculate the above parameters (Fig. 1). In brief, outer wall and lumen areas at the reference points were averages of the proximal and distal outer wall and lumen areas. Wall area was calculated by subtracting the lumen area from the outer wall area. The calculations for PA, SR and PPB were as follows: $\mathrm{PA}=$ wall area at MLN site - wall area at the reference site, $\mathrm{SR}=1-\mathrm{lm}$ area at MLN site/ lumen area at the reference site, $\mathrm{PPB}=\mathrm{PA}$ /outer wall area at MLN site. For the Self-referenced measurement method, the MLN site was used to as the reference. $\mathrm{PA}=$ inner wall area at MLN site - lumen area at MLN site, SR = PA/ inner wall area at MLN site, $\mathrm{PPB}=\mathrm{PA}$ /outer wall area at MLN site (Fig. 1). The distance between proximal and distal reference locations was calculated and recorded (distance $=m$ * Slice thickness $+(m-1) *$ Gap, $m$ indicates the number of slices between both locations). To measure the intra-observer variability, BA plaque was measured twice at two different time points that were separated by a 2 -week interval to avoid any recall bias.

\section{Statistical analysis}

All statistical analyses were performed using the SPSS software for Windows (version 16.0, SPSS Inc., Chicago, IL, USA) and MedCalc (version 13.0.0.0, MedCalc Software, Mariakerke, Belgium). A Kolmogorov-Smirnov test was used to test for normal distribution. Quantitative data were described as the means \pm standard deviation. Qualitative data were expressed as count (percentage). Statistically significant differences between the Selfreferenced and Referenced measurement methods and between the repeated measurements of PA, SR and PPB with both methods were assessed using a two-tailed paired t-test. The coefficient of variation $(\mathrm{CV})$ was calculated by determining the standard deviation (SD) of the two paired measurements of $\mathrm{T} 2 \mathrm{~W}$ images and dividing the mean of those measurements. The intraclass correlation coefficient (ICC) with a two-way mixed model and single consistency type was determined and used to evaluate the agreement between the repeat measurements. According to Shout and Fleiss [29], values of $\mathrm{ICC}<0.4$ represent poor agreement,
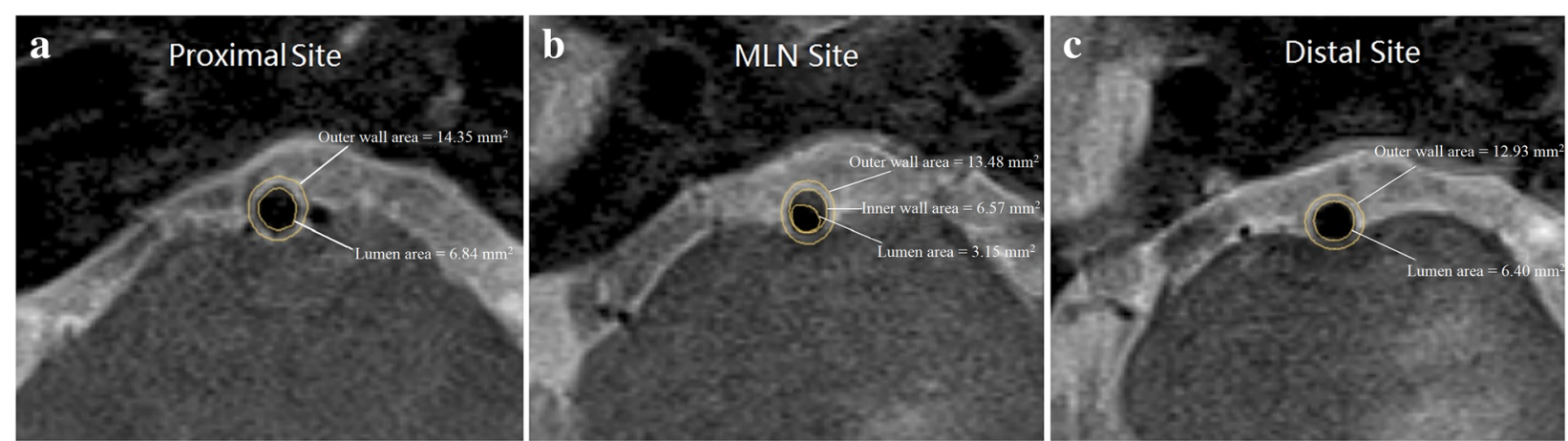

Fig. 2 Examples of outer wall areas, inner wall areas and lumen areas at the proximal sites (a), maximal lumen narrowing (MLN) sites (b) and distal sites (c) that were traced manually in a 53-year-old female patient with basilar atherosclerotic plaque. For the Self-referenced measurement method, the plaque area is $3.42 \mathrm{~mm}^{2}$, stenosis rate is 0.521 , and percent plaque burden is 0.254 . For the Referenced measurement method, the outer wall area at the reference site is $13.64 \mathrm{~mm}^{2}$, the lumen area at reference site is $6.62 \mathrm{~mm}^{2}$, the wall area at the reference site is $7.02 \mathrm{~mm}^{2}$, the wall area at the MLN site is $10.33 \mathrm{~mm}^{2}$, the plaque area is $3.31 \mathrm{~mm}^{2}$, the stenosis rate is 0.551 , and the percent plaque burden is 0.246 
Table 1 Patient demographics

\begin{tabular}{ll}
\hline Variable & $n=37$ \\
\hline Male sex & $28(75.7 \%)$ \\
Age (years) & $62.4 \pm 10.1$ \\
Body mass index & $24.4 \pm 3.1$ \\
Hypertension & $27(73.0 \%)$ \\
$\quad$ Systolic pressure(mmHg) & $159.0 \pm 26.5$ \\
Diastolic pressure (mmHg) & $94.8 \pm 17.0$ \\
Diabetes mellitus & $17(45.9 \%)$ \\
Fasting blood-glucose (mg/dL) & $8.3 \pm 9.7$ \\
Hyperlipidaemia & $19(51.4 \%)$ \\
$\quad$ Total cholesterol (mmol/L) & $4.08 \pm 1.05$ \\
Triglyceride (mmol/L) & $1.41 \pm 0.53$ \\
High-density lipoprotein (mmol/L) & $1.06 \pm 0.19$ \\
$\quad$ Low density lipoprotein (mmol/L) & $2.52 \pm 0.95$ \\
Stenosis rate & $0.69 \pm 0.18$ \\
Apolipoprotein A1 (g/L) & $1.14 \pm 0.23$ \\
Apolipoprotein B (g/L) & $0.83 \pm 0.19$ \\
Smoking & $16(43.2 \%)$ \\
\hline
\end{tabular}

0.4 to 0.75 represent good agreement, and $>0.75$ represent excellent agreement. Bland-Altman plots were also derived for those measurements [30] and bias and limits of agreement were calculated. A $P$ value of $<0.05$ was considered as statistically significant.

\section{Results}

While all patients completed HRMRI examinations, three patients were excluded in the final analysis because of poor image quality. The scores of images quality were as follows: 2 in 5 patients, 3 in 21 patients and 4 in 11 patients. Thus, thirty-seven patients $(28$ males and 9 females, $47-79$ years old, mean age $=62$ years) were included in the study, and quantitative analysis was performed with their data. The patient demographics were presented in Table 1 . The time interval between the stroke event and plaque imaging was also recorded, and the average time was $16.2 \pm 8.2$ days. The distances between proximal and distal reference locations were $11.12 \pm 3.78 \mathrm{~mm}$.

\section{Comparison of self-referenced and referenced measurement methods}

The results of the Self-referenced and Referenced measurement methods are presented in Table 2. PA determined by both measurement methods were $9.015 \pm$ $4.916 \mathrm{~mm}^{2}$ and $8.678 \pm 4.634 \mathrm{~mm}^{2}$, respectively. The SR values were $0.699 \pm 0.172$ and $0.688 \pm 0.184$ for the Selfreferenced and Referenced methods, respectively. In addition, the PPB values of the two measurement methods were $0.375 \pm 0.111$ and $0.361 \pm 0.117$, respectively. The bias and limit of agreement measurements using Bland-Altman plots for the PA, SR and PPB were $0.336(-2.018,2.690), 0.011(-0.072,0.094)$ and 0.014 $(-0.083,0.110)$ (Fig. 3), respectively. There were no statistically significant differences between the two methods in those measurements ( $p$-values $>0.05)$.

\section{Reproducibility of the self-referenced measurement method}

The results for the Self-referenced measurement reproducibility analysis are presented in Table 3. Repeated measurements with the Self-referenced method were $8.954 \pm 4.833 \mathrm{~mm}^{2}$ and $9.071 \pm 5.023 \mathrm{~mm}^{2}$ for PA, $0.697 \pm 0.173$ and $0.695 \pm 0.172$ for SR, and $0.374 \pm 0.112$ and $0.372 \pm 0.112$ for PPB. No statistically significant differences were observed for those measurements $(p$ values $>0.05$ ). The results indicate an excellent reproducibility for the repeated measurements, with the ICC and $\mathrm{CV}$ values ranging from 0.976 to 0.990 and 3.73 to $5.61 \%$, respectively. Excellent agreement was also observed in the repeated measurement reproducibility analysis employing Bland-Altman plots (Fig. 4).

\section{Reproducibility of the referenced measurement method}

The results for the Referenced measurement reproducibility are presented in Table 4 . The repeated measurements of the Referenced method were $8.569 \pm 4.444$ $\mathrm{mm}^{2}$ and $8.783 \pm 4.892 \mathrm{~mm}^{2}$ for PA, $0.686 \pm 0.185$ and $0.684 \pm 0.186$ for SR, and $0.361 \pm 0.112$ and $0.361 \pm 0.117$ for PPB. No statistically significant differences were observed for those measurements ( $\mathrm{p}$-values $>0.05$ ). The results indicate an excellent reproducibility for the repeated measurements, with the ICC and CV values ranging from 0.928 to 0.971 and 4.64 to $9.87 \%$, respectively. Excellent agreement was also observed in the

Table 2 Comparison of Self-referenced and Referenced measurement methods in assessing basilar plaque

\begin{tabular}{llllll}
\hline & Self-referenced (Mean \pm SD) & Referenced (Mean \pm SD) & Bias & LoA & $p$ \\
\hline Plaque area $\left(\mathrm{mm}^{2}\right)$ & $9.015 \pm 4.916$ & $8.678 \pm 4.634$ & 0.336 & $(-2.018,2.690)$ & 0.097 \\
Stenosis rate & $0.699 \pm 0.172$ & $0.688 \pm 0.184$ & 0.011 & $(-0.072,0.094)$ & 0.121 \\
Percent plaque burden & $0.375 \pm 0.111$ & $0.361 \pm 0.117$ & 0.014 & $(-0.083,0.110)$ & 0.761 \\
\hline
\end{tabular}

SD Standard deviation, LoA Limit of Agreement 

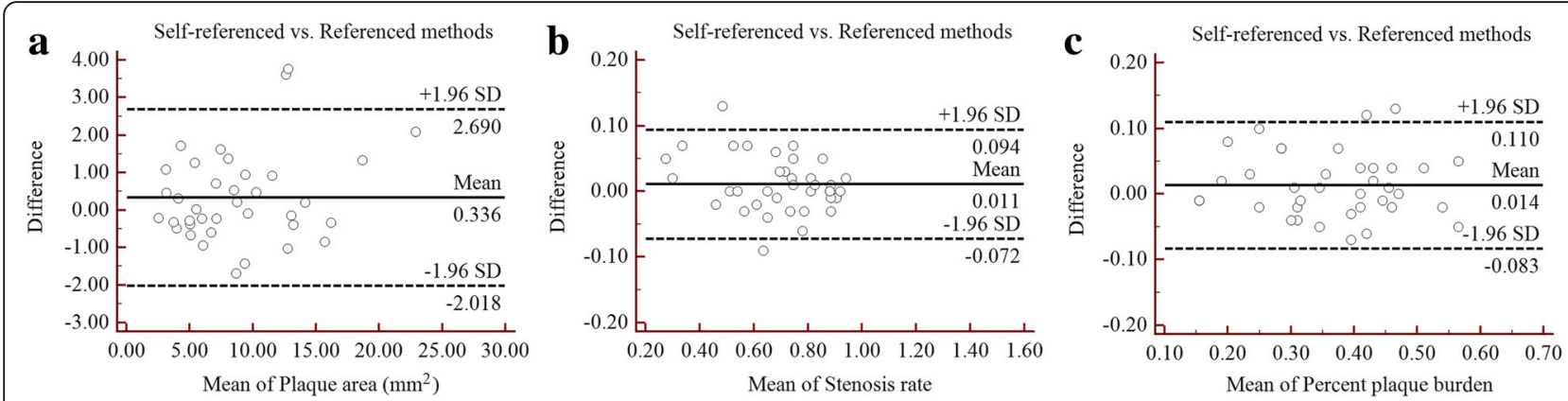

Fig. 3 Comparison of the Self-referenced and Referenced measurement methods for BA plaque using Bland-Altman plots, plaque area (a), stenosis rate (b) and percent plaque burden (c)

repeated measurement reproducibility analysis employing Bland-Altman plots (Fig. 5).

\section{Discussion}

The present study evaluated the Self-referenced and Referenced measurement methods in assessing basilar atherosclerotic plaque employing dark blood HRMRI at 3 Tesla. The results show no significant differences between the Self-referenced and Referenced measurement. In addition, repeated measurements of PA, SR and PPB demonstrate excellent reproducibility in evaluating basilar atherosclerotic plaque.

This study employed Self-referenced and Referenced measurement methods to assess basilar atherosclerotic plaque and compared both techniques in evaluating morphologic parameters of the basilar artery. For the Self-referenced measurement method, the MLN site was used as the reference site to assess several parameters such as PA, SR and PPB. It is a simple and fast method to evaluate those parameters compared with the Referenced measurement method, in which both the nearest normal segments that are proximal and distal to the MLN site were used as the reference. Our data indicate that there were no significant difference between both methods in assessing the PA, SR and PPB of basilar plaque. In addition, only a small degree of bias was observed between the Selfreferenced and Referenced measurement methods using Bland-Altman plots (Fig. 3). In addition, there was excellent reproducibility in the measurements of PA, SR and PPB by both methods, with ICC and CV values ranging from 0.941 to 0.990 and 3.73 to $9.87 \%$, respectively. The reliability of these methods is of significant importance for the evaluation of basilar atherosclerotic plaque morphologic parameters. Briefly, the Self-referenced measurement method is a suitable alternative to the Referenced measurement method in quantifying basilar plaque morphology.

Dark blood HRMRI has been increasingly used to evaluate the morphology of basilar atherosclerotic plaque, as it offers good boundary outlines for the vessel-blood and the vessel-cerebral spine fluid borders [25, 31-33]. Kim et al. found that evaluation of stenosis associated with basilar atherosclerotic plaque using HRMRI is more accurate than evaluation using magnetic resonance angiography. The study involved a large sample size (219 patients) compared with other studies, and the Self-referenced measurement method was used to assess the degree of stenosis [23]. The discrepancy between HRMRI and magnetic resonance angiography was more obvious with mild stenosis, which shows that HRMRI is more sensitive for evaluating the early phase of intracranial atherosclerosis [23]. Ma et al. investigated the morphology of advanced basilar atherosclerotic plaque using the Referenced measurement method [26], Zhu et al. also used this method to assess the morphologic characteristics of atherosclerotic middle cerebral arteries using HRMRI at 3 Tesla [24] and Feng et al. explored arterial remodelling of basilar atherosclerosis in pontine infarction [25]. However, sites that were proximal and distal to the MLN were first selected, and then

Table 3 Reproducibility of Self-referenced measurement method in assessing basilar plaque

\begin{tabular}{|c|c|c|c|c|c|}
\hline & $\begin{array}{l}\text { Measurement } 1 \\
\text { (Mean } \pm \text { SD) }\end{array}$ & Measurement 2 (Mean \pm SD) & ICC $(95 \% \mathrm{Cl})$ & CV (\%) & $p$ \\
\hline Plaque area $\left(\mathrm{mm}^{2}\right)$ & $8.954 \pm 4.833$ & $9.071 \pm 5.023$ & $0.990(0.981-0.995)$ & 5.61 & 0.329 \\
\hline Stenosis rate & $0.697 \pm 0.173$ & $0.695 \pm 0.172$ & $0.977(0.955-0.988)$ & 3.73 & 0.759 \\
\hline Percent plaque burden & $0.374 \pm 0.112$ & $0.372 \pm 0.112$ & $0.976(0.954-0.988)$ & 4.59 & 0.549 \\
\hline
\end{tabular}

SD Standard deviation, ICC Intra-class coefficient, CI Confidence interval, CV Coefficient of variability 

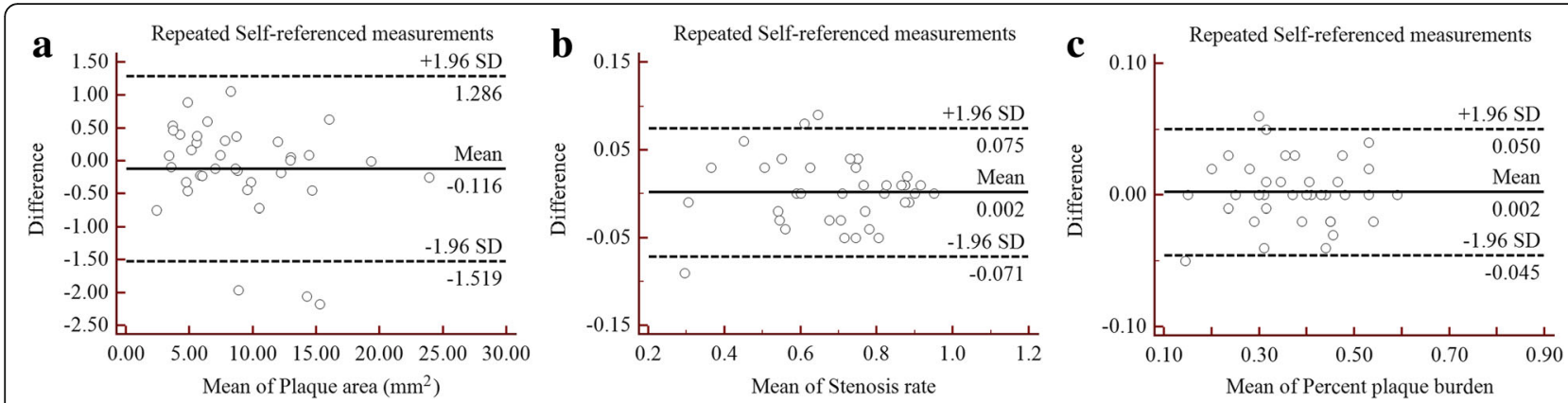

Fig. 4 Bland-Altman plots of reproducibility for the Self-referenced method, plaque area (a), stenosis rate (b) and percent plaque burden (c) of BA plaques were evaluated using the Self-referenced measurement method

PA, SR and PPB were evaluated using the Referenced measurement method in those studies. In contrast, the Self-referenced measurement method, only needed the MLN site to calculate those parameters. In short, the main differences between the Self-referenced measurement method and the Referenced measurement method are presented as follows: (1) Only one site (MLN) was used in Self-referenced measurement method, while three sites (proximal, MLN and distal) were needed in the Referenced measurement method. (2) For plaque area quantification, the former method is more simple and rapid, as there is no need to calculate the reference outer wall, lumen and wall areas, which are needed in the latter method. Therefore, the Self-referenced method is a simple and convenient method for assessing plaque morphologic characteristics compared with the Referenced method. Our comparison of the Self-referenced and Referenced measurement method in evaluating basilar atherosclerotic plaque morphology demonstrates an excellent agreement between both the methods in assessment of PA, SR and PPB parameters, which indicates that the Self-referenced measurement method can be used in the clinical setting.

Imaging modalities including transcranial doppler ultrasound, computed tomography angiography and magnetic resonance angiography have been used to assess intracranial atherosclerosis. However, while those imaging techniques offer an evaluation of the vascular lumen, they are incapable of providing vessel wall information [23]. More importantly, the vessel wall characteristics can help us better understand the pathophysiology of atherosclerosis, which has a significant effect on patient management [4]. Dark blood HRMRI can delicately delineate plaque presence and morphology and provide new insights into atherosclerotic burden [2]. To increase the black blood effect, saturation band, inversion recovery, motion-sensitized driven-equilibrium, and delay alternating with nutation for tailored excitation are the most commonly used techniques during the magnetization preparation phase [34-37]. Because of the inherent flow void effect of the TSE protocol and due to its simplicity and low specific absorption ratio properties, the saturation band was used to saturate the inflow blood signal when imaging basilar plaque. Black blood high-resolution T2W images were used to obtain quantitative measurements because of the good contrast that was obtained between the lumen and plaque, the vessel wall and cerebrospinal fluid compared to the other imaging protocols [38].

The present study has several limitations. First, the sample size is relatively small. Further studies with a larger sample size are needed to validate the present results. Second, the Self-referenced measurement method could not be directly used to calculate the remodelling patterns of basilar atherosclerotic plaque, such as positive and negative remodelling patterns. However, we can use the Referenced measurement method to calculate those patterns if needed. Lastly, the outer wall, inner wall and lumen boundaries on the T2W images were

Table 4 Reproducibility of Referenced measurement method in assessing basilar plaque

\begin{tabular}{|c|c|c|c|c|c|}
\hline & $\begin{array}{l}\text { Measurement } 1 \\
\text { (Mean } \pm \text { SD) }\end{array}$ & Measurement 2 (Mean \pm SD) & ICC (95\% Cl) & CV (\%) & $p$ \\
\hline Plaque area $\left(\mathrm{mm}^{2}\right)$ & $8.569 \pm 4.444$ & $8.783 \pm 4.892$ & $0.971(0.944-0.985)$ & 9.87 & 0.289 \\
\hline Stenosis rate & $0.686 \pm 0.185$ & $0.684 \pm 0.186$ & $0.970(0.942-0.985)$ & 4.64 & 0.774 \\
\hline Percent plaque burden & $0.361 \pm 0.112$ & $0.361 \pm 0.117$ & $0.941(0.887-0.969)$ & 7.64 & 0.967 \\
\hline
\end{tabular}

SD Standard deviation, ICC Intra-class coefficient, CI Confidence interval, CV Coefficient of variability 

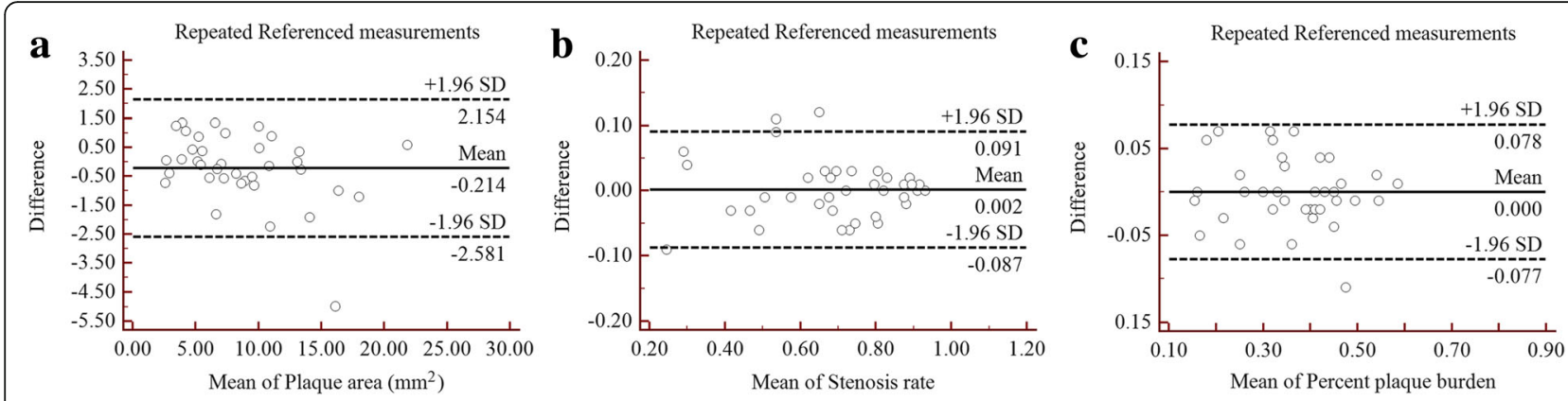

Fig. 5 Bland-Altman plots of reproducibility for the Referenced methods, plaque area (a), stenosis rate (b) and percent plaque burden (c) of BA plaques were evaluated using the Referenced measurement method

manually outlined to assess the PA, SR and PPB of basilar atherosclerotic plaque. An automatic plaque measurement tool is needed, as this may improve efficiency and reduce errors between the repeated measurements.

\section{Conclusions}

In conclusion, the Self-referenced and Referenced methods are slightly equivalent and both reliable, however, the former is quicker and easier and it may serve as a promising method for evaluation of basilar atherosclerotic plaque.

\section{Abbreviations}

3D TOF-MRA: Three-dimensional time-of-flight magnetic resonance angiography; BA: Basilar artery; CV: Coefficient of variation; FOV: Field of view; HRMRI: High-resolution magnetic resonance imaging; ICC: Intraclass correlation coefficient; MLN: Maximal lumen narrowing; PA: Plaque area; PPB: Percent plaque burden; SD: Standard deviation; SR: Stenosis rate; TA: Acquisition time; TIA: Transient ischaemic attack; TR/TE: Repetition time/ echo time

\section{Acknowledgements}

Thanks for the International Society for Magnetic Resonance in Medicine (ISMRM) committee to accepted this work as a traditional poster in 2017 (https://www.ismrm.org/17/TraditionalPoster.pdf, NO. 2456).

\section{Funding}

This work was supported by the national key clinical specialist construction programs of China (Grant Number N/A) and the National Natural Science Foundation of China (Grant Number 31470910, 31600755).

\section{Availability of data and materials}

The datasets used and/or analyzed during the current study are available from the corresponding author on reasonable request.

\section{Authors' contributions}

$J$, and QL conceived of the present idea. LC and ZQ designed the study. Data acquisition was performed by LC. LC and WP performed the statistical analysis. TS, QZ and WP contributed to the data analysis and interpretation. LC was contributed to writing the manuscript. All the authors read and approved the final manuscript.

\section{Ethics approval and consent to participate}

This study was approved by the Committee on Ethics of Biomedical Research, Changhai Hospital of Shanghai. Written informed consent to participate in this study was obtained from each patient.

\section{Consent for publication}

Written informed consent was obtained from each patient to publish.

\section{Competing interests}

The authors declare that they have no conflict of interest.

\section{Publisher's Note}

Springer Nature remains neutral with regard to jurisdictional claims in published maps and institutional affiliations.

Received: 23 August 2018 Accepted: 16 November 2018

Published online: 03 December 2018

\section{References}

1. El Aidi H, Mani V, Weinshelbaum KB, Aguiar SH, Taniguchi H, Postley JE, et al. Cross-sectional, prospective study of MRI reproducibility in the assessment of plaque burden of the carotid arteries and aorta. Nat Clin Pract Cardiovasc Med. 2009;6:219-28.

2. Dong L, Wang J, Yarnykh VL, Underhill HR, Neradilek MB, Polissar N, et al. Efficient flow suppressed MRI improves interscan reproducibility of carotid atherosclerosis plaque burden measurements. J Magn Reson Imaging. 2010; 32:452-8

3. Suh DC, Lee SH, Kim KR, Park ST, Lim SM, Kim SJ, et al. Pattern of atherosclerotic carotid stenosis in Korean patients with stroke: different involvement of intracranial versus extracranial vessels. AJNR Am J Neuroradiol. 2003:24:239-44

4. Huang B, Yang WQ, Liu XT, Liu HJ, Li PJ, Lu HK. Basilar artery atherosclerotic plaques distribution in symptomatic patients: a 3.0T high-resolution MR study. Eur J Radiol. 2013;82:e199-203.

5. Vidal A, Bureau Y, Wade T, Spence JD, Rutt BK, Fenster A, et al. Scan-rescan and intra-observer variability of magnetic resonance imaging of carotid atherosclerosis at 1.5 T and 3.0 T. Phys Med Biol. 2008:53:6821-35.

6. Mossa-Basha M, Shibata DK, Hallam DK, de Havenon A, Hippe DS, Becker KJ, et al. Added value of Vessel Wall magnetic resonance imaging for differentiation of nonocclusive intracranial Vasculopathies. Stroke. 2017;48: 3026-33.

7. Harteveld AA, van der Kolk AG, van der Worp HB, Dieleman N, Zwanenburg JJM, Luijten PR, et al. Detecting intracranial Vessel Wall lesions with 7Tmagnetic resonance imaging: patients with posterior circulation ischemia versus healthy controls. Stroke. 2017:48:2601-4.

8. Yang WJ, Wong KS, Chen XY. Intracranial atherosclerosis: from microscopy to high-resolution magnetic resonance imaging. J Stroke. 2017;19:249-60.

9. Kim JM, Jung $\mathrm{KH}$, Sohn $\mathrm{CH}$, Moon J, Shin JH, Park J, et al. Intracranial plaque enhancement from high resolution vessel wall magnetic resonance imaging predicts stroke recurrence. Int J Stroke. 2016;11:171-9.

10. Wu F, Song H, Ma Q, Xiao J, Jiang T, Huang $X$, et al. Hyperintense plaque on intracranial Vessel Wall magnetic resonance imaging as a predictor of artery-to-artery embolic infarction. Stroke. 2018;49:905-11.

11. Alkhalil M, Edmond E, Edgar L, Digby JE, Omar O, Robson MD, et al. The relationship of perivascular adipose tissue and atherosclerosis in the aorta and carotid arteries, determined by magnetic resonance imaging. Diab Vasc Dis Res. 2018. https://www doi.org/10.1177/1479164118757923.

12. Watase H, Sun J, Hippe DS, Balu N, Li F, Zhao X, et al. Carotid artery remodeling is segment specific: an in vivo study by Vessel Wall magnetic resonance imaging. Arterioscler Thromb Vasc Biol. 2018;38:927-34. 
13. Lu M, Peng P, Cui Y, Qiao H, Li D, Cai J, et al. Association of Progression of carotid Artery Wall volume and recurrent transient ischemic attack or stroke: a magnetic resonance imaging study. Stroke. 2018;49:614-20.

14. Zhao X, Hippe DS, Li R, Canton GM, Sui B, Song Y, et al. Prevalence and characteristics of carotid artery high-risk atherosclerotic plaques in Chinese patients with cerebrovascular symptoms: a Chinese atherosclerosis risk evaluation II study. J Am Heart Assoc. 2017;6.

15. Lin R, Chen S, Liu G, Xue Y, Zhao X. Association between carotid atherosclerotic plaque calcification and Intraplaque hemorrhage: a magnetic resonance imaging study. Arterioscler Thromb Vasc Biol. 2017;37:1228-33.

16. Abd-Elmoniem KZ, Ramos N, Yazdani SK, Ghanem AM, Holland SM, Freeman AF, et al. Coronary atherosclerosis and dilation in hyper lgE syndrome patients: depiction by magnetic resonance vessel wall imaging and pathological correlation. Atherosclerosis. 2017;258:20-5

17. Dweck MR, Puntman V, Vesey AT, Fayad ZA, Nagel E. MR imaging of coronary arteries and plaques. JACC Cardiovasc Imaging. 2016;9:306-16.

18. Makowski MR, Henningsson M, Spuentrup E, Kim WY, Maintz D, Manning WJ, et al. Characterization of coronary atherosclerosis by magnetic resonance imaging. Circulation. 2013;128:1244-55.

19. Xie G, Zhang N, Xie Y, Nguyen C, Deng Z, Bi X, et al. DANTE-prepared threedimensional FLASH: a fast isotropic-resolution MR approach to morphological evaluation of the peripheral arterial wall at 3 tesla. J Magn Reson Imaging. 2016:43:343-51

20. Langham MC, Desjardins B, Englund EK, Mohler ER, 3rd, Floyd TF, Wehrli FW. Rapid high-resolution, self-registered, dual lumen-contrast MRI method for vessel-wall assessment in peripheral artery disease:: A Preliminary Investigation Acad Radiol 2016;23:457-467.

21. Isbell DC, Meyer CH, Rogers WJ, Epstein FH, DiMaria JM, Harthun NL, et al. Reproducibility and reliability of atherosclerotic plaque volume measurements in peripheral arterial disease with cardiovascular magnetic resonance. J Cardiovasc Magn Reson. 2007;9:71-6.

22. Zhou C, Qiao H, He L, Yuan C, Chen H, Zhang Q, et al. Characterization of atherosclerotic disease in thoracic aorta: a 3D, multicontrast vessel wall imaging study. Eur J Radiol. 2016;85:2030-5.

23. Kim YS, Lim SH, Oh KW, Kim JY, Koh SH, Kim J, et al. The advantage of highresolution MRI in evaluating basilar plaques: a comparison study with MRA. Atherosclerosis. 2012;224:411-6.

24. Zhu XJ, Du B, Lou X, Hui FK, Ma L, Zheng BW, et al. Morphologic characteristics of atherosclerotic middle cerebral arteries on 3T highresolution MRI. AJNR Am J Neuroradiol. 2013;34:1717-22.

25. Feng C, Hua T, Xu Y, Liu XY, Huang J. Arterial remodeling of basilar atherosclerosis in isolated pontine infarction. Neurol Sci. 2015;36:547-51.

26. Ma N, Jiang WJ, Lou X, Ma L, Du B, Cai JF, et al. Arterial remodeling of advanced basilar atherosclerosis: a 3-tesla MRI study. Neurology. 2010;75: 253-8.

27. Chen L, Liu Q, Zhan Q, Zhang X, Lu J. Comparison of two different measurement methods in evaluating basilar atherosclerotic plaque using high resolution MRI at 3T. Honolulu: International Society for Magnetic Resonance in Medicine; 2017.

28. Underhill HR, Yarnykh VL, Hatsukami TS, Wang J, Balu N, Hayes CE, et al. Carotid plaque morphology and composition: initial comparison between 1. 5- and 3.0-T magnetic field strengths. Radiology. 2008;248:550-60.

29. Shrout PE, Fleiss JL. Intraclass correlations: uses in assessing rater reliability. Psychol Bull. 1979;86:420-8.

30. Bland JM, Altman DG. Statistical methods for assessing agreement between two methods of clinical measurement. Lancet. 1986;1:307-10.

31. Lim SH, Choi H, Kim HT, Kim J, Heo SH, Chang Dl, et al. Basilar plaque on high-resolution MRI predicts progressive motor deficits after pontine infarction. Atherosclerosis. 2015:240:278-83.

32. Ryu CW, Kwak HS, Jahng GH, Lee HN. High-resolution MRI of intracranial atherosclerotic disease. Neurointervention. 2014:9:9-20.

33. Cai JM, Hatsukami TS, Ferguson MS, Small R, Polissar NL, Yuan C. Classification of human carotid atherosclerotic lesions with in vivo multicontrast magnetic resonance imaging. Circulation. 2002;106:1368-73.

34. Felmlee JP, Ehman RL. Spatial presaturation: a method for suppressing flow artifacts and improving depiction of vascular anatomy in MR imaging. Radiology. 1987;164:559-64.

35. Li L, Miller KL, Jezzard P. DANTE-prepared pulse trains: a novel approach to motion-sensitized and motion-suppressed quantitative magnetic resonance imaging. Magn Reson Med. 2012;68:1423-38.
36. Wang J, Yarnykh VL, Hatsukami T, Chu B, Balu N, Yuan C. Improved suppression of plaque-mimicking artifacts in black-blood carotid atherosclerosis imaging using a multislice motion-sensitized drivenequilibrium (MSDE) turbo spin-echo (TSE) sequence. Magn Reson Med. 2007:58:973-81.

37. Yarnykh VL, Yuan C. Multislice double inversion-recovery black-blood imaging with simultaneous slice reinversion. J Magn Reson Imaging. 2003; 17:478-83.

38. Yang WQ, Huang B, Liu XT, Liu HJ, Li PJ, Zhu WZ. Reproducibility of highresolution MRI for the middle cerebral artery plaque at $3 T$. Eur J Radiol. 2014;83:e49-55.

\section{Ready to submit your research? Choose BMC and benefit from:}

- fast, convenient online submission

- thorough peer review by experienced researchers in your field

- rapid publication on acceptance

- support for research data, including large and complex data types

- gold Open Access which fosters wider collaboration and increased citations

- maximum visibility for your research: over $100 \mathrm{M}$ website views per year

At $\mathrm{BMC}$, research is always in progress.

Learn more biomedcentral.com/submissions 